\title{
Graphene-based membranes for organic solvent nanofiltration
}

\author{
Ruirui $\mathrm{Hu}$ and Hongwei $\mathrm{Zhu}{ }^{*}$
}

Different from the conventional polymeric separation membranes having a wide flexible-pore size distribution, layered graphene-based films with interconnected nanochannels provide narrowly well-defined nanopore-size distribution and thus are extremely advantageous for precise liquid filtration and separation. Recently, considerable progress has been achieved in graphene-based membranes for molecular sieving in water. For example, it was found that sub-micrometer-thick graphene oxide (GO) membranes were almost impermeable to liquids, vapors, or gases but allowed unimpeded permeation of water [1] because of the nearly frictionless flow of water molecules through the two-dimensional hydrophobic nonoxidized capillaries. Moreover, it was further demonstrated that GO membranes could act as molecular sieves to block all solutes with hydrated radii $>0.45 \mathrm{~nm}$ when immersed in water [2], in which size exclusion appears to account for this behavior. For smaller sizefitted solutes that can enter in nanochannels, GO membrane could achieve more accurate ion selectivity via the diverse interactions between ions and $\mathrm{sp}^{2}$ clusters or oxygen-containing functional groups on GO basal planes [3].

The findings mentioned above implicate that GO membranes are extremely promising for a broad spectrum of separation due to their tunability in pore size to remove various target solutes, including expansion by inserting large spacers and decrease by reducing, crosslinking, etc. [4]. Consequently, most existing studies focused on the adjustment of pore structure for high flux and retention. The representative novel nanostrandchanneled GO membrane exhibited 10-time higher permeance than the conventional polymeric separation membranes [5]. However, graphene-based membranes confronted with some crucial challenges in practical industrial applications, such as scale-up of membranes to large-area, the narrow interlayer spacing in water for highly effective desalination, molecular transport mechanism confined in nanocapillaries, stability in water for long periods of time, especially graphene-based membranes used for organic solvent nanofiltration (OSN).

Over the past two years, some successes have been achieved in these aspects. Large-area GO membranes were accessible by an industrially adaptable method of blade coating, which aligned the discotic nematic phase of GO on a porous support [6]. The highly ordered GO membranes formed organized channels and demonstrated outstanding water permeability. Another alternative method is spray coating GO aqueous dispersion and few-layered graphene to yield robust membranes that are easy and scalable to produce [7]. The hybrid layered membranes maintained effective $\mathrm{NaCl}$ rejection near $85 \%$ and $96 \%$ for an anionic dye. The achievement of $97 \%$ high rejection for $\mathrm{NaCl}$ is a significant breakthrough due to the controllable limited swelling of GO laminates in water [8]. The ion permeation rates were found to decrease exponentially with decreasing sieve size because of dehydration effects whereas water transport was slightly affected. By the cationic control, the angstrom precision of interlayer spacing was further achieved and maintained against the swelling of GO membrane in aqueous solution [9]. As a result, the exceptional high desalination of more than $99 \%$ was reached. As for the water transport mechanism within atomic-scale capillaries, the unexpectedly fast flow (up to $1 \mathrm{~m} \mathrm{~s}^{-1}$ ) was achieved, which was attributed to the enhanced structural order, high capillary pressure, and large slip length [10]. To improve graphene-based membranes for a prolonged period operation, polyvinyl alcohol interfacial adhesive layer was introduced between graphene and support membrane, which dramatically contributed to a steady performance for up to $120 \mathrm{~h}$ under intense cross-flow [7].

State Key Lab of New Ceramics and Fine Processing, School of Materials Science and Engineering, Tsinghua University, Beijing 100084, China

*Email: hongweizhu@tsinghua.edu.cn 

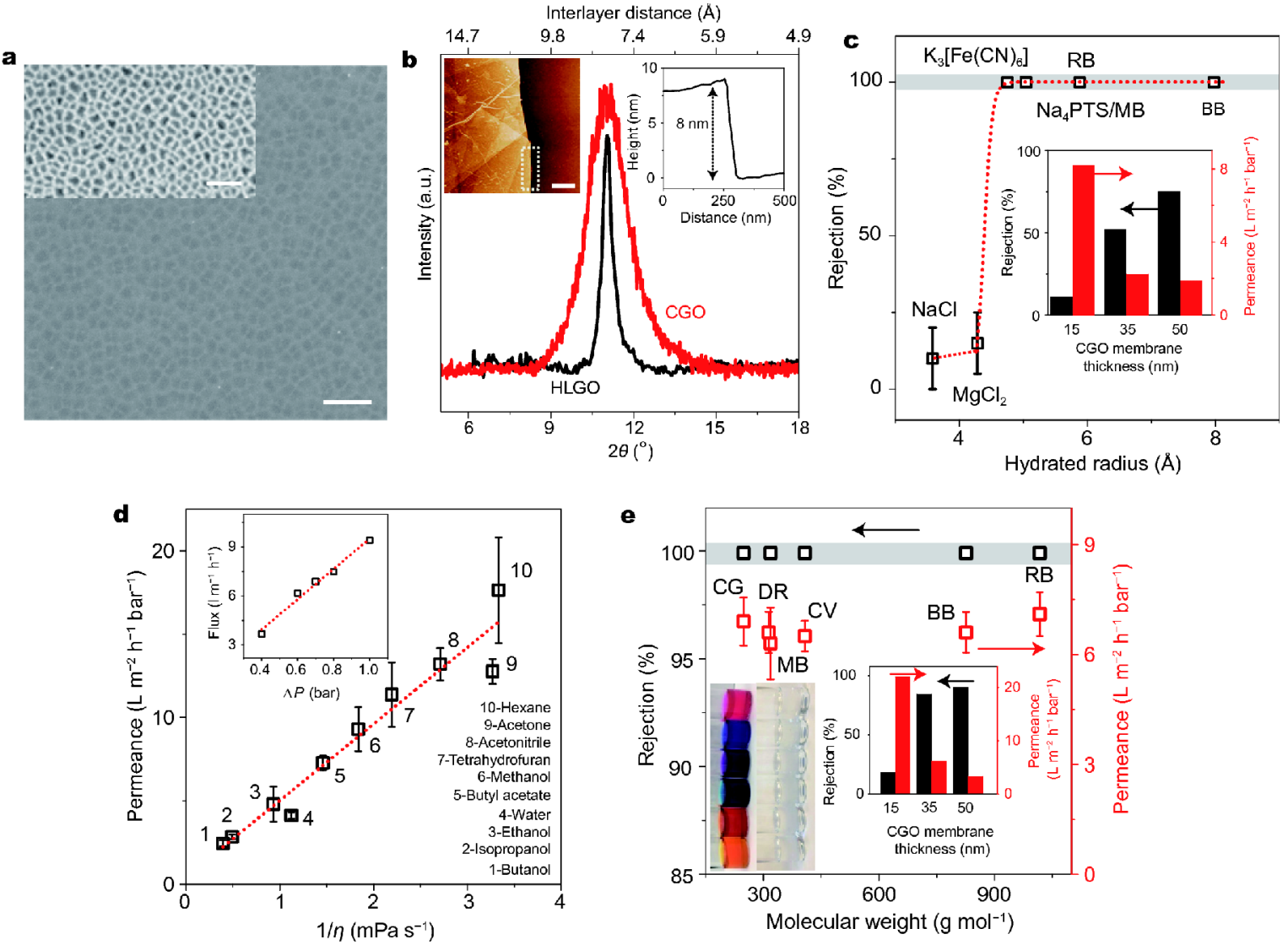

Hydrated distance $(A)$
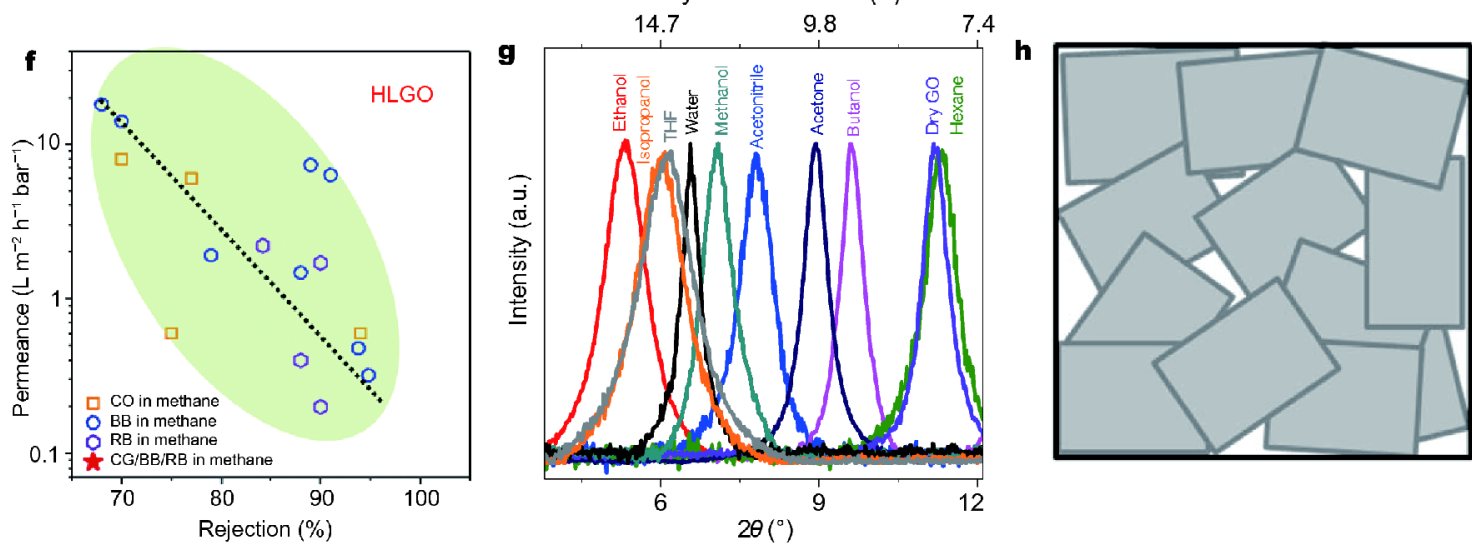

Figure 1 (a) SEM image of ultrathin HLGO membrane on the anodisc alumina support. Scale bar, $1 \mu \mathrm{m}$. Inset: SEM image of the pristine support surface. Scale bar: $500 \mathrm{~nm}$. (b) XRD spectra of HLGO and CGO membranes. Left inset: AFM image of HLGO film. Scale bar: $500 \mathrm{~nm}$. Right inset: AFM height profiles. (c) Molecular sieving properties of HLGO membranes to various solutes. Inset: the filtration performance of CGO membranes to MB aqueous solution. (d) Permeance properties of HLGO membranes to water and various organic solvents as a function of their viscosity. Inset: the effect of pressure gradient on methanol permeance. (e) Filtration performance of HLGO membranes with several dyes in methanol. Left inset: photographs of dyes in methanol before and after filtration. Right inset: filtration results of CGO membranes. (f) Comparison of dyes filtration in an organic solvent using HLGO membrane with reported OSN membranes. (g) XRD spectra of 70-nm-thick HLGO membrane in diverse organic solvents. (h) Schematic diagram of randomly distributed pinholes interconnected by graphene sheets. Reprinted with permission from Ref. [11], Copyright 2017, Nature Publishing Group. 
As considerable progress has been achieved for GO laminates in water, the exploration of graphene-based laminates for OSN is of great significance due to the instability of polymeric membranes in an organic solvent, yet has been lacking because of the impermeability of organic solvent through sub-micrometer-thick GO membranes. Recently, Nair group optimized GO laminar structure and demonstrated the potential of ultrathin GO membranes for OSN by achieving $>99.9 \%$ rejection of small organic dyes in methanol [11], thus opening up a new world of filtration and separation for graphene-based membranes.

The ultrathin GO membrane was prepared by vacuum filtration of large GO flake suspension (lateral size of 10-20 $\mu \mathrm{m}$ ) on an Anodisc alumina support (Fig. 1a). The 8-nm-thick GO membrane showed a narrow XRD peak compared with conventional GO (CGO, smaller lateral size of $0.1-0.6 \mu \mathrm{m}$ ), suggesting the presence of higher interlayer alignment (Fig. 1b). The molecular sieving performance of this highly laminated GO membrane (HLGO) was assessed using an aqueous solution consisting of several salts and large molecules (Fig. 1c). The ultrathin HLGO membranes exhibited an ultra-sharp sieving cut-off by blocking all ions with hydrated radii larger than $0.45 \mathrm{~nm}$. To probe the liquid permeance of HLGO membranes, the filtration of water and various organic solvents was performed (Fig. 1d). Surprisingly, the ultrathin HLGO membranes were highly permeable to all tested solvents, and the linear dependence of permeance on $1 / \eta$ was revealed. The highest permeance was observed for hexane with the lowest viscosity. The potential of HLGO membrane for OSN was evaluated by investigating several dyes in methanol solutions. The filtration results showed nearly $100 \%$ dye rejection with only $10-30 \%$ decrease in solvent permeance (Fig. 1e), which is remarkable for OSN. The outstanding performance of HLGO membrane with simultaneous high retention and fast permeation was demonstrated to be superior to previously reported OSN membranes (Fig. 1f). To elucidate the mechanism of organic solvent permeation, the interlayer spacing of HLGO membrane in different organic solvents was analyzed by XRD (Fig. 1g). The results indicated that polar solvents intercalated into GO layers while nonpolar ones did not produce any impact. This excluded the possibility that organic molecules were transported through HLGO membranes from the interlayer capillaries. Therefore, a permeation pathway involving pinholes was proposed. As shown in Fig.
$1 \mathrm{~h}$, pinholes are originated from random staking of individual GO sheets. Molecular transport is expected to begin with rapidly filling the pinholes, followed by the migration from one pinhole to another, where the interlayer diffusion provides atomic-scale sieving.

Graphene-based membranes will continue to attract tremendous attention in materials science community because of their unique ultrafast transport accompanied with precise molecular sieving properties in both water and organic filtration applications. From the perspective of water purification, the stability of graphene-based membranes in water for long periods of time up to several months remains a challenge. To achieve efficient OSN separation, the design and fabrication of sub-micrometerthick membranes with disordered laminar structure and smaller interlayer distance are required.

Received 8 December 2017; accepted 12 December 2017;

published online 8 January 2018

1 Nair RR, Wu HA, Jayaram PN, et al. Unimpeded permeation of water through helium-leak-tight graphene-based membranes. Science, 2012, 335: 442-444

2 Joshi RK, Carbone P, Wang FC, et al. Precise and ultrafast molecular sieving through graphene oxide membranes. Science, 2014, 343: 752-754

3 Sun P, Zheng F, Zhu M, et al. Selective trans-membrane transport of alkali and alkaline earth cations through graphene oxide membranes based on cation $-\pi$ interactions. ACS Nano, 2014, 8: 850-859

4 Mi B. Graphene oxide membranes for ionic and molecular sieving. Science, 2014, 343: 740-742

5 Huang $\mathrm{H}$, Song Z, Wei N, et al. Ultrafast viscous water flow through nanostrand-channelled graphene oxide membranes. Nat Commun, 2013, 4: 2979

6 Akbari A, Sheath P, Martin ST, et al. Large-area graphene-based nanofiltration membranes by shear alignment of discotic nematic liquid crystals of graphene oxide. Nat Commun, 2016, 7: 10891

7 Morelos-Gomez A, Cruz-Silva R, Muramatsu H, et al. Effective $\mathrm{NaCl}$ and dye rejection of hybrid graphene oxide/graphene layered membranes. Nat Nanotechnol, 2017, 12: 1083-1088

8 Abraham J, Vasu KS, Williams CD, et al. Tunable sieving of ions using graphene oxide membranes. Nat Nanotechnol, 2017, 12: 546-550

9 Chen L, Shi G, Shen J, et al. Ion sieving in graphene oxide membranes via cationic control of interlayer spacing. Nature, 2017, 550: $380-383$

10 Radha B, Esfandiar A, Wang FC, et al. Molecular transport through capillaries made with atomic-scale precision. Nature, 2016, 538: 222-225

11 Yang Q, Su Y, Chi C, et al. Ultrathin graphene-based membrane with precise molecular sieving and ultrafast solvent permeation. Nat Mater, 2017, 16: 1198-1202 